\title{
On some generalization of box splines
}

\author{
by Zygmunt Wronicz (Kraków)
}

\begin{abstract}
We give a generalization of box splines. We prove some of their properties and we give applications to interpolation and approximation of functions.

1. Introduction. An extended complete Chebyshev system $U_{n}=\left\{u_{i}\right\}_{i=0}^{n}$ of functions of class $C^{n}$ in the interval $I=[a, b]$ is a generalization of the system $\left\{t^{i}\right\}_{i=0}^{n}$ of power functions. We can write such a system in the canonical form (see $[9,12])$

$$
\begin{aligned}
& u_{0}(t)=w_{0}(t), \\
& u_{i}(t)=w_{0}(t) \int_{a}^{t} w_{1}\left(\tau_{1}\right) \int_{a}^{\tau_{1}} w_{2}\left(\tau_{2}\right) \ldots \int_{a}^{\tau_{i-1}} w_{i}\left(\tau_{i}\right) d \tau_{i} \ldots d \tau_{1},
\end{aligned}
$$

$i=1, \ldots, n$, where $w_{j} \in C^{n-j}(I), w_{j}(t)>0$ for $t \in I, j=0, \ldots, n$.

For $u_{j}(t)=(t-a)^{j}, j=0, \ldots, n$, we have $w_{0}=1, w_{i}=i, i=1, \ldots, n$.

To generalize box splines we shall use a similar method to that used to obtain (1). Box splines are used in approximation theory, in interpolation of functions, in the finite element variational method, in the theory of wavelets and in other branches of mathematics (see $[3,5,7,8,10]$ ). Not only algebraic splines but also Chebyshevian splines play an important role in approximation theory. We give a generalization of box splines and a few of their main properties; we call the new splines Chebyshevian box splines. Then we define a fundamental function and give some applications of it to interpolation and approximation of functions.
\end{abstract}

2. Chebyshevian box splines. We need some notation and definitions. We say that an integer $n \times s$ matrix $V_{s}=\left\{v_{1}, \ldots, v_{s}\right\}, v_{i} \in \mathbb{Z}^{n} \backslash\{0\}$,

\footnotetext{
1991 Mathematics Subject Classification: 41A15, 41A63, 41A05, 41A25.

Key words and phrases: box splines, Chebyshevian splines, interpolation, approximation.
} 
$i=1, \ldots, s$, is admissible if $\operatorname{rank} V_{n}=n$ (the first $n$ columns of $V_{s}$ are linearly independent), $s \geq n$. Below we assume that the matrix $V_{s}$ is admissible.

Define

We see that

$$
\left\langle V_{s}\right\rangle=\left\{\sum_{j=1}^{s} t_{j} v_{j}: 0 \leq t_{j} \leq 1, j=1, \ldots, s\right\} .
$$

$$
\left\langle V_{s}\right\rangle=V_{s}\left([0,1]^{s}\right)
$$

Let $W=\left\{w_{1}, \ldots, w_{s-n}\right\}$ be a sequence of continuous functions on $\mathbb{R}^{n}$ such that

(i) each $w_{j}$ is periodic, i.e. $w_{j}(x+\alpha)=w_{j}(x)$ for $\alpha \in \mathbb{Z}^{n}$,

(ii) $0<a_{j} \leq w_{j}(x) \leq b_{j}<\infty$ for $x \in \mathbb{R}^{n}$, where $a_{j}$ and $b_{j}$ are some constants.

Definition 1. The box spline $B\left(x \mid V_{s}, W\right)$ with respect to $V_{s}$ and $W$ is defined as follows:

$$
B\left(x \mid V_{n}, W\right)=\frac{\chi_{\left\langle V_{n}\right\rangle}(x)}{\left|\operatorname{det} V_{n}\right|},
$$

where $\chi_{\left\langle V_{n}\right\rangle}$ is the characteristic function of the set $\left\langle V_{n}\right\rangle$ and

$$
\begin{aligned}
B & \left(x \mid V_{n+k}, W\right) \\
& =\int_{0}^{1} w_{k}\left(x-t v_{n+k}\right) B\left(x-t v_{n+k} \mid V_{n+k-1}, W\right) d t, \quad k=1, \ldots, s-n .
\end{aligned}
$$

For $w_{j}=1, j=1, \ldots, s-n$, we obtain algebraic box splines, denoted by $B\left(x \mid V_{s}\right)$ (see $\left.[1,2,4,6]\right)$.

Theorem 1. For any function $f \in C\left(\mathbb{R}^{n}\right)$ we have

$$
\int_{\mathbb{R}^{n}} f(x) B\left(x \mid V_{n}, W\right) d x=\int_{[0,1]^{n}} f\left(V_{n} u\right) d u,
$$

$$
\begin{aligned}
\int_{\mathbb{R}^{n}} f(x) & B\left(x \mid V_{s}, W\right) d x \\
= & \int_{[0,1]^{s}} f\left(V_{s} u\right) w_{s-n}\left(\widetilde{V}_{s} u\right) w_{s-n-1}\left(\widetilde{V}_{s-1} u\right) \ldots w_{1}\left(\widetilde{V}_{n+1} u\right) d u,
\end{aligned}
$$

where $\widetilde{V}_{n+k}=\widetilde{V}_{s, n+k}=\left[v_{1}, \ldots, v_{n+k-1}, 0, \ldots, 0\right]$ with $s-n-k+1$ zeros, $u=\left[u_{1}, \ldots, u_{s}\right], d u=d u_{1} \ldots d u_{s}$.

P r o of (by induction on $s$ ). For $s=n$ we change variables in the integral (see [6])

$$
\int_{\mathbb{R}^{n}} f(x) B\left(x \mid V_{n}, W\right) d x=\int_{\left\langle V_{n}\right\rangle} f(x) \frac{\chi\left\langle V_{n}\right\rangle}{\left|\operatorname{det} V_{n}\right|} d x=\int_{[0,1]^{n}} f\left(V_{n} u\right) d u
$$


because $V_{n}\left([0,1]^{n}\right)=\left\langle V_{n}\right\rangle$. Assume that (3) holds true for $s=n+k$. Then

$$
\begin{array}{rl}
\int_{\mathbb{R}^{n}} & f(x) B\left(x \mid V_{n+k+1}, W\right) d x \\
= & \int_{0 \mathbb{R}^{n}}^{1} f(x) w_{k+1}\left(x-t v_{n+k+1}\right) B\left(x-t v_{n+k+1} \mid V_{n+k}, W\right) d x d t \\
= & \int_{0 \mathbb{R}^{n}}^{1} f\left(x+t v_{n+k+1}\right) w_{k+1}(x) B\left(x \mid V_{n+k}, W\right) d x d t \\
= & \int_{0[0,1]^{n+k}}^{1} f\left(V_{n+k} u+t v_{n+k+1}\right) w_{k+1}\left(V_{n+k} u\right) w_{k}\left(\widetilde{V}_{n+k} u\right) \ldots w_{1}\left(\widetilde{V}_{n+1} u\right) d u d t \\
= & \int_{[0,1]^{n+k+1}} f\left(V_{n+k+1} y\right) w_{k+1}\left(\widetilde{V}_{n+k+1} y\right) w_{k}\left(\widetilde{V}_{n+k} y\right) \ldots w_{1}\left(\widetilde{V}_{n+1} y\right) d y
\end{array}
$$

and we have proved (3) for $s=n+k+1$.

Assume that $\int_{[0,1]^{s}} w_{s-n}\left(\widetilde{V}_{s-n} u\right) \ldots w_{1}\left(\widetilde{V}_{n+1} u\right) d u=1$. Putting $f=1$ we obtain

$$
\int_{\mathbb{R}^{n}} B\left(x \mid V_{s}, W\right) d x=1 .
$$

Lemma 1. Let $v \in \mathbb{R}^{n} \backslash\{0\}$ and the function $G: \mathbb{R}^{n} \rightarrow \mathbb{R}$ be continuous on its convex support $D$. Then the function

$$
F(x)=\int_{0}^{1} G(x-t v) d t
$$

is continuous on its support

$$
\operatorname{supp} F=\{x:[x, x-v] \cap D \neq \emptyset\} .
$$

Pr o of. Since $D$ is convex, the supports of the functions $g_{1}(t)=G(y-t v)$ and $g(t)=G(x-t v)$ are segments whose lengths depend continuously on $y$ and $x$ from $G$. Hence by continuity of $G$ we obtain $\varliminf_{y \rightarrow x} F(y)=F(x)$.

COROllary. The function $B\left(x \mid V_{s}, W\right)$ is continuous on its support $\left\langle V_{s}\right\rangle$.

Theorem 2 (cf. [6]). Let $C$ be a nonsingular integer $n \times n$ matrix. Then

$$
B\left(x \mid C V_{s}, W\right)=\frac{1}{|\operatorname{det} C|} B\left(C^{-1} x \mid V_{s}, \widetilde{W}\right),
$$

where $\widetilde{W}=\left\{\widetilde{w}_{1}, \ldots, \widetilde{w}_{s-n}\right\}, \widetilde{w}_{j}(x)=w_{j}(C x), j=1, \ldots, s-n$. 
Proof. Let $f \in C\left(\mathbb{R}^{n}\right)$. Applying Theorem 1 we have

$$
\begin{array}{rl}
\int_{\mathbb{R}^{n}} & f(x) B\left(x \mid C V_{s}, W\right) d x \\
& =\int_{[0,1]^{s}} f\left(C V_{s} u\right) w_{s-n}\left(\widetilde{C V}_{s} u\right) w_{s-n-1}\left(\widetilde{C V}_{s-1} u\right) \ldots w_{1}\left(\widetilde{C V}_{n+1} u\right) d u \\
& =\int_{[0,1]^{s}} f\left(C V_{s} u\right) w_{s-n}\left(C \widetilde{V}_{s} u\right) w_{s-n-1}\left(C \widetilde{V}_{s-1} u\right) \ldots w_{1}\left(C \widetilde{V}_{n+1} u\right) d u .
\end{array}
$$

Putting $g(x)=f(C x)$ and applying Theorem 1 again we obtain

$$
\begin{aligned}
\int_{\mathbb{R}^{n}} f(x) B(x \mid & \left.C V_{s}, W\right) d x \\
& =\int_{[0,1]^{s}} g\left(V_{s} u\right) \widetilde{w}_{s-n}\left(\widetilde{V}_{s} u\right) \widetilde{w}_{s-n-1}\left(\widetilde{V}_{s-1} u\right) \ldots \widetilde{w}_{1}\left(\widetilde{V}_{n+1} u\right) d u \\
& =\int_{\mathbb{R}^{n}} f(C x) B\left(x \mid V_{s}, \widetilde{W}\right) d x=\int_{\mathbb{R}^{n}} f(x) \frac{B\left(C^{-1} x \mid V_{s}, \widetilde{W}\right)}{|\operatorname{det} C|} d x .
\end{aligned}
$$

Since the function $f$ was chosen arbitrarily and $B\left(x \mid V_{s}, W\right)$ is continuous (Corollary of Theorem 1) we have proved (4).

Theorem 3. Let

$$
D_{v_{s}, w_{s-n}} f(x)=\frac{1}{w_{s-n}(x)} \lim _{t \rightarrow 0+} \frac{1}{t}\left[f\left(x+t v_{s}\right)-f(x)\right] .
$$

Then

$$
D_{v_{s}, w_{s-n}} B\left(x \mid V_{s}, W\right)=B\left(x \mid V_{s-1}, W\right)-B\left(x-v_{s} \mid V_{s-1}, W\right)
$$

at every point of continuity of $B\left(x \mid V_{s-1}, W\right)$.

Proof (cf. [6]). Let $g(x)=w_{s-n}(x) B\left(x \mid V_{s-1}, W\right)$. Then

$$
\begin{aligned}
w_{s-n}(x) D_{v_{s} \cdot w_{s-n}} B & \left(x \mid V_{s}, W\right) \\
& =\lim _{t \rightarrow 0+} \frac{1}{t}\left\{\int_{0}^{1} g\left[x-(r-t) v_{s}\right] d r-\int_{0}^{1} g\left(x-r v_{s}\right) d r\right\} \\
& =\lim _{t \rightarrow 0+} \frac{1}{t}\left[\int_{-t}^{1-t} g\left(x-r v_{s}\right) d r-\int_{0}^{1} g\left(x-r v_{s}\right) d r\right] \\
& =\lim _{t \rightarrow 0+} \frac{1}{t}\left[\int_{-t}^{0} g\left(x-r v_{s}\right) d r-\int_{1-t}^{1} g\left(x-r v_{s}\right) d r\right]
\end{aligned}
$$




$$
\begin{aligned}
& =\lim _{t \rightarrow 0+} \frac{1}{t}\left[\int_{0}^{t} g\left(x+r v_{s}\right) d r-\int_{0}^{t} g\left(x+r v_{s}-v_{s}\right) d r\right] \\
& =w_{s-n}(x) B\left(x \mid V_{s-1}, W\right)-w_{s-n}\left(x-v_{s}\right) B\left(x-v_{s} \mid V_{s-1}, W\right) \\
& =w_{s-n}(x)\left[B\left(x \mid V_{s-1}, W\right)-B\left(x-v_{s} \mid V_{s-1}, W\right)\right]
\end{aligned}
$$

and we have got (5).

\section{A fundamental function. Let}

$$
G_{V_{s}}=\bigcup_{i_{1}<\ldots<i_{n-1}}\left\langle v_{i_{1}, \ldots,} v_{i_{n-1}}\right\rangle+\left\{\sum_{j \neq i_{1}, \ldots, i_{n-1}}^{s} \varepsilon_{j} v_{j}: \varepsilon_{j}=0,1\right\} \quad \text { for } n>1
$$

and

$$
G_{V_{s}}=\left\{\sum_{j=1}^{s} \varepsilon_{j} v_{j}: \varepsilon_{j}=0,1\right\} \quad \text { for } n=1 .
$$

As in the algebraic case we can prove that in the set $\mathbb{R}^{n} \backslash G_{V_{s}}$,

$$
D_{v_{n, 1}} D_{v_{n+1}, w_{1}} \ldots D_{v_{s}, w_{s-n}} B\left(x \mid V_{s}, W\right)=0 .
$$

Now we may consider the space $S\left(V_{s}, W\right)$ spanned by the integer translates of the box spline $B\left(x \mid V_{s}, W\right)$ :

$$
S\left(V_{s}, W\right)=\operatorname{span}\left\{B\left(x-\alpha \mid V_{s}, W\right): \alpha \in \mathbb{Z}^{n}\right\} .
$$

For $n=1$ the space $S\left(V_{s}, W\right)$ is included in the space of cardinal Chebyshevian splines. In the algebraic case for $n=1$ we have cardinal B-splines

$$
\begin{aligned}
N_{1}(x) & =\chi_{[0,1)}(x), \\
N_{m}(x) & =\left(N_{m-1} * N_{1}\right)(x)=\int_{0}^{1} N_{m-1}(x-t) d t, \quad m \geq 2 .
\end{aligned}
$$

Now we need some definitions, lemmas and theorems.

DeFinition 2. The family of columns of the matrix $V_{s}$ is called unimodular if the first $n$ columns are linearly independent and $\forall_{Y \subset V_{s}, \sharp Y=n}|\operatorname{det} Y| \leq 1$.

Let $\widehat{f}$ denote the Fourier transform of $f$, i.e.

$$
\widehat{f}(x)=\int_{\mathbb{R}^{n}} f(t) e^{-2 \pi i t \cdot x} d t .
$$

In the algebraic case we have the following

Lemma 2 (see $[4,6]$ ). The family $V=V_{s}$ is unimodular if and only if

$$
\left\{x \in \mathbb{R}^{n}: \forall_{\alpha \in \mathbb{Z}^{n}} \widehat{B}(x-\alpha \mid V)=0\right\}=\emptyset .
$$

For Chebyshevian box splines the condition (6) may not hold for a family which is not unimodular: 
EXAMPLE.

$$
\begin{array}{ccc}
V_{4}=\left[\begin{array}{cccc}
1 & 0 & 2 & 0 \\
0 & 1 & 0 & 1
\end{array}\right], & & w_{1}=1, \\
w_{2}\left(x_{1}, x_{2}\right)=e^{-\left|2 x_{1}-1\right|} & & \text { for } 0 \leq x_{1} \leq 1, \\
w_{2}\left(x_{1}+\alpha, x_{2}\right)=w_{2}\left(x_{1}, x_{2}\right) & & \text { for } \alpha \in \mathbb{Z} .
\end{array}
$$

Applying (3) we obtain

$$
\begin{aligned}
\widehat{B}(x \mid & \left.V_{4}, W\right) \\
& =\int_{[0,1]^{4}} e^{-2 \pi i\left[\left(u_{1}+2 u_{3}\right) x_{1}+\left(u_{2}+u_{4}\right) x_{2}\right]} w_{2}\left(u_{1}+2 u_{3}, u_{2}\right) d u_{1} d u_{2} d u_{3} d u_{4} \\
& =\left(\frac{1-e^{-2 \pi i x_{2}}}{2 \pi i x_{2}}\right)^{2} \sum_{k=1}^{8} \iint_{D_{k}} e^{-2 \pi i\left(u_{1}+2 u_{3}\right) x_{1}} g_{k}\left(u_{1}, u_{3}\right) d u_{1} d u_{3},
\end{aligned}
$$

where

$$
\begin{aligned}
D_{1} & =\left\{\left(u_{1}, u_{3}\right): 0 \leq u_{1} \leq 1 / 2,0 \leq u_{3} \leq 1 / 4-u_{1} / 2\right\}, \\
D_{2} & =\left\{\left(u_{1}, u_{3}\right): 0 \leq u_{1} \leq 1 / 2,1 / 4-u_{1} / 2 \leq u_{3} \leq 1 / 2-u_{1} / 2\right\}, \\
D_{3} & =\left\{\left(u_{1}, u_{3}\right): 1 / 2 \leq u_{1} \leq 1,0 \leq u_{3} \leq 1 / 2-u_{1} / 2\right\}, \\
D_{4} & =\left\{\left(u_{1}, u_{3}\right): 0 \leq u_{1} \leq 1,1 / 2-u_{1} / 2 \leq u_{3} \leq 3 / 4-u_{1} / 2\right\}, \\
D_{5} & =\left\{\left(u_{1}, u_{3}\right): 0 \leq u_{1} \leq 1,3 / 4-u_{1} / 2 \leq u_{3} \leq 1-u_{1} / 2\right\}, \\
D_{6} & =\left\{\left(u_{1}, u_{3}\right): 0 \leq u_{1} \leq 1 / 2,1-u_{1} / 2 \leq u_{3} \leq 1\right\}, \\
D_{7} & =\left\{\left(u_{1}, u_{3}\right): 1 / 2 \leq u_{1} \leq 1,1-u_{1} / 2 \leq u_{3} \leq 5 / 4-u_{1} / 2\right\}, \\
D_{8} & =\left\{\left(u_{1}, u_{3}\right): 1 / 2 \leq u_{1} \leq 1,5 / 4-u_{1} / 2 \leq u_{3} \leq 1\right\}, \\
g_{1} & =e^{-1+2 u_{1}+4 u_{3}}, \quad g_{2}=g_{3}=e^{1-2 u_{1}-4 u_{3}}, \quad g_{4}=e^{-3+2 u_{1}+4 u_{3}}, \\
g_{5} & =e^{3-2 u_{1}-4 u_{3}}, \quad g_{6}=g_{7}=e^{-5+2 u_{1}+4 u_{3}}, \quad g_{8}=e^{5-2 u_{1}-4 u_{3}} .
\end{aligned}
$$

Hence

where

$$
\widehat{B}\left(x \mid V_{4}, W\right)=\left(\frac{1-e^{-2 \pi i x_{2}}}{2 \pi i x_{2}}\right)^{2} \frac{g\left(x_{1}\right)}{8 e\left(1+\pi^{2} x_{1}^{2}\right)^{2}},
$$

$$
\begin{aligned}
g\left(x_{1}\right)= & \left(1+2 e \cdot e^{-\pi i x_{1}}-5 e^{-2 \pi i x_{1}}+4 e \cdot e^{-3 \pi i x_{1}}-5 e^{-4 \pi i x_{1}}\right. \\
& \left.+2 e \cdot e^{-5 \pi i x_{1}}+e^{-6 \pi i x_{1}}\right) \\
& +2 \pi i\left(1-2 e \cdot e^{-\pi i x_{1}}+e^{-2 \pi i x_{1}}-e^{-4 \pi i x_{1}}\right. \\
& \left.+2 e \cdot e^{-5 \pi i x_{1}}-e^{-6 \pi i x_{1}}\right) x_{1} \\
& +\pi^{2}\left(-1+2 e \cdot e^{-\pi i x_{1}}-3 e^{-2 \pi i x_{1}}+4 e \cdot e^{-3 \pi i x_{1}}-3 e^{-4 \pi i x_{1}}\right. \\
& \left.+2 e \cdot e^{-5 \pi i x_{1}}-e^{-6 \pi i x_{1}}\right) x_{1}^{2} \\
= & \sum_{k=0}^{2} \alpha_{k}\left(e^{\pi i x_{1}}\right) x_{1}^{k}
\end{aligned}
$$


and the coefficients $\alpha_{k}$ depend only on $e^{\pi i x_{1}}$. If $g\left(\widetilde{x}_{1}\right)=0$, then $g\left(\widetilde{x}_{1}+2 j\right)=$ $\sum_{k=0}^{2} \alpha_{k}\left(e^{\pi i \widetilde{x}_{1}}\right)\left(\widetilde{x}_{1}+2 j\right)^{k}$ and since it is a polynomial of degree 2 with respect to $j$, it is different from zero for some $j$ except for the case when all the coefficients of $g$ are zero, and this happens for $x_{1}=1 / 2$ and $x_{1}=3 / 2$. Hence the condition (6) is not satisfied.

Let $X=V \cup-V=\left\{v_{1}, \ldots, v_{s},-v_{1}, \ldots,-v_{s}\right\}$. Then we define

$$
\begin{aligned}
\widetilde{B}(x \mid X, W) & =\left(B(\cdot \mid V, W) * B\left(\cdot \mid-V, W^{-}\right)\right)(x) \\
& =\int_{\mathbb{R}^{n}} B(x-t \mid V, W) B\left(t \mid-V, W^{-}\right) d t .
\end{aligned}
$$

LEMMA 3. We have

$$
B(-x \mid V, W)=B\left(x \mid-V, W^{-}\right), \quad \widetilde{B}(x \mid X, W)=\widetilde{B}(-x \mid X, W)
$$

and

$$
\widehat{B}\left(x \mid-V, W^{-}\right)=\overline{\widehat{B}(x \mid V, W)},
$$

where $W^{-}=\{f: f(-x) \in W$ and $-V=\{x \in V:-x \in V\}$.

The proof follows directly from the definition of box splines.

Lemma 4. Let $D_{V_{s}, W} f=D_{v_{n+1}, w_{1}} \ldots D_{v_{s}, w_{s-n}} f$ and let $B\left(x \mid V_{s}\right)$ and $B(x \mid X)$ be the algebraic box splines with respect to $V_{s}$ and $X=V_{s} \cup-V_{s}$ respectively; moreover, in the cases $W=1$ let all the functions $w_{j}$ be equal to one. Then

$$
D_{-V_{s}, W^{-}} D_{V_{s}, W} \widetilde{B}(x \mid X, W)=D_{-V_{s}, 1} D_{V_{s}, 1} B(x \mid X) .
$$

$\mathrm{Pr}$ o of. Using the fact that convolution is commutative and Theorem 3 we obtain the equality

$$
\begin{aligned}
D_{-V_{s}, W^{-}} & D_{V_{s}, W} \widetilde{B}(x \mid X, W) \\
& =\int_{\mathbb{R}^{n}} D_{V_{s}, W} B\left(x-t \mid V_{s}, W\right) D_{-V_{s}, W^{-}} B\left(t \mid-V_{s}, W^{-}\right) d t \\
& =\int_{\mathbb{R}^{n}} D_{V_{s}, 1} B\left(x-t \mid V_{s}\right) D_{-V_{s}, 1} B\left(t \mid-V_{s}\right) d t \\
& =D_{-V_{s}, 1} D_{V_{s} 1} \int_{\mathbb{R}^{n}} B\left(x-t \mid V_{s}\right) B\left(t \mid-V_{s}\right) d t=D_{-V_{s}, 1} D_{V_{s}, 1} B(x \mid X)
\end{aligned}
$$

since $B\left(x \mid V_{s}\right) * B\left(x \mid-V_{s}\right)=B(x, X)$ (see $\left.[4,6]\right)$ and we have proved the lemma.

Corollary. $\widetilde{B}(x \mid X, W)=B(x \mid \widetilde{V}, \widetilde{W})$, where

$$
\begin{aligned}
\widetilde{V} & =\left\{v_{1}, \ldots, v_{n},-v_{1}, \ldots,-v_{n},-v_{n+1}, \ldots,-v_{s},-v_{n+1}, \ldots,-v_{s}\right\}, \\
\widetilde{W} & =\{\underbrace{1, \ldots, 1}_{n}, w_{1}(x), \ldots, w_{s-n}(x), w_{1}(-x), \ldots, w_{s-n}(-x)\} .
\end{aligned}
$$


LEMma 5. Let $f, \widehat{f} \in C(\mathbb{R})$ and for some $\delta>0$,

$$
\begin{array}{ll}
|f(x)| \leq C(1+\|x\|)^{-n-\delta}, & x \in \mathbb{R}^{n}, \\
|\widehat{f}(x)| \leq C(1+\|x\|)^{-n-\delta}, & x \in \mathbb{R}^{n} .
\end{array}
$$

Then for every $x \in \mathbb{R}^{n}$,

$$
\sum_{\alpha \in \mathbb{Z}^{n}} f(x-\alpha)=\sum_{\alpha \in \mathbb{Z}^{n}} \widehat{f}(\alpha) e^{2 \pi i \alpha \cdot x}
$$

For the proof we refer to [11] (see also [6]).

LEMMA 6. Let functions $\varphi$ and $\psi$ be integrable, bounded and with compact support. Then for every $x \in \mathbb{R}^{n}$,

$$
\sum_{\alpha \in \mathbb{Z}^{n}} \widehat{\varphi}(x-\alpha) \widehat{\psi}(x-\alpha)=\sum_{\alpha \in \mathbb{Z}^{n}}(\varphi * \psi)(-\alpha) e^{2 \pi i \alpha \cdot x} .
$$

For the proof we refer to [6].

TheOREm 4. Let the family $V$ be admissible and satisfy (6). Then for every $x \in \mathbb{R}^{n}$,

$$
P_{X, W}(x)=\sum_{\alpha \in \mathbb{Z}^{n}} \widetilde{B}(\alpha \mid X, W) e^{2 \pi i \alpha \cdot x} \neq 0 .
$$

Pro of (cf. [6]). We apply Lemma 6 to the functions $\varphi(x)=B(x \mid V, W)$ and $\psi(x)=B\left(x \mid-V, W^{-}\right)$. Hence

$$
\begin{aligned}
\sum_{\alpha \in \mathbb{Z}^{n}}[B(\cdot \mid V, W)]^{\wedge}(x-\alpha)\left[B\left(\cdot \mid-V, W^{-}\right)\right]^{\wedge}(x-\alpha) & \\
& =\sum_{\alpha \in \mathbb{Z}^{n}}\left(B(\cdot \mid V, W) * B\left(\cdot \mid-V, W^{-}\right)\right)(-\alpha) e^{2 \pi i \alpha \cdot x} \\
& =\sum_{\alpha \in \mathbb{Z}^{n}} \widetilde{B}(-\alpha \mid X, W) e^{2 \pi i \alpha \cdot x}=\sum_{\alpha \in \mathbb{Z}^{n}} \widetilde{B}(\alpha \mid X, W) e^{2 \pi i \alpha \cdot x} .
\end{aligned}
$$

Using Lemma 3 we obtain

$$
[B(\cdot \mid V, W)]^{\wedge}(x)\left[B\left(\cdot \mid-V, W^{-}\right)\right]^{\wedge}(x)=\left|[B(\cdot \mid V, W)]^{\wedge}(x)\right|^{2} .
$$

Hence

$$
\sum_{\alpha \in \mathbb{Z}^{n}}\left|[B(\cdot \mid V, W)]^{\wedge}(x-\alpha)\right|^{2}=\sum_{\alpha \in \mathbb{Z}^{n}} \widetilde{B}(\alpha \mid X, W) e^{2 \pi i \alpha \cdot x}
$$

and by (6) we obtain the theorem.

Since the trigonometric polynomial $P_{X, W}$ is different from zero for every $x \in \mathbb{R}^{n}$, the function $1 / P_{X, W}$ is periodic and of class $C^{\infty}$. Hence we may expand it in a Fourier series:

$$
\frac{1}{P_{X, W}(x)}=\sum_{\alpha \in \mathbb{Z}^{n}} b_{X, W}(\alpha) e^{2 \pi i \alpha \cdot x}
$$


where

$$
b_{X, W}(\alpha)=\int_{[0,1]^{n}} \frac{1}{P_{X, W}(x)} e^{-2 \pi i \alpha \cdot x} d x .
$$

Lemma 7. Let the family $V$ be admissible and satisfy (6). Then the sequence $b_{X, W}=\left\{b_{X, W}(\alpha)\right\}$ of coefficients of the expansion of $1 / P_{X, W}$ is exponentially decaying, i.e. there exist constants $C>0$ and $0<q<1$ such that

$$
\left|b_{X, W}(\alpha)\right| \leq C q^{\|\alpha\|}, \quad \alpha \in \mathbb{Z}^{n},
$$

where $\|\alpha\|=\left|\alpha_{1}\right|+\ldots+\left|\alpha_{n}\right|$.

The proof is the same as in the algebraic case (see [6]).

Let $V$ satisfy the condition (6).

DeFinition 3. The fundamental function associated with the family $X=$ $V \cup-V$ and the family $W$ is the function

$$
\Phi_{X, W}(x)=\sum_{\alpha \in \mathbb{Z}^{n}} b_{X, W}(\alpha) \widetilde{B}(x-\alpha \mid X, W) .
$$

Lemma 8. For every $\alpha \in \mathbb{Z}^{n}$,

$$
\Phi_{X, W}(\alpha)=\delta_{0, \alpha} .
$$

Proof (see [6]). Taking the Cauchy product of the Fourier series for $P_{X, W}$ and $1 / P_{X, W}$ we obtain $(7)$.

As in the algebraic case we obtain (see [6])

Lemma 9. There exist constants $C>0$ and $0<q<1$ such that

$$
\left|\Phi_{X, W}(x)\right| \leq C q^{\|x\|}, \quad x \in \mathbb{R}^{n} .
$$

Now we may define interpolating operators $I$ and $I_{h}$ as follows: for every function $g$ defined on $\mathbb{Z}^{n}$ we put

$$
\begin{gathered}
I g(x)=\sum_{\alpha \in \mathbb{Z}^{n}} g(\alpha) \Phi_{X, W}(x-\alpha), \\
I_{h} g(x)=\sum_{\alpha \in \mathbb{Z}^{n}} g(\alpha) \Phi_{X, W}(x / h-\alpha) .
\end{gathered}
$$

The problem of the convergence of the operators $I_{h}$ will be considered in another paper.

Let

$$
B_{V, W}^{*}(x)=\sum_{\alpha \in \mathbb{Z}^{n}} b_{X, W}(\alpha) B(x-\alpha \mid V, W), \quad x \in \mathbb{R}^{n} .
$$

We have the following (see [6]) 
Lemma 10. For every $\beta \in \mathbb{Z}^{n}$,

$$
\left(B_{V, W}^{*}, B(\cdot-\beta \mid V, W)\right)_{\mathbb{R}^{n}}=\delta_{0, \beta} .
$$

Moreover, there exist constants $C>0$ and $0<q<1$ such that

$$
\left|B_{V, W}^{*}(x)\right| \leq C q^{\|x\|}, \quad x \in \mathbb{R}^{n} .
$$

P r o of. Applying Lemma 3 and changing variables we obtain $\left(B_{X, W}^{*}, B(\cdot-\beta \mid V, W)\right)_{\mathbb{R}^{n}}$

$$
\begin{aligned}
& =\sum_{\alpha \in \mathbb{Z}^{n}} b_{X, W}(\alpha) \int_{\mathbb{R}^{n}} B(x-\alpha \mid V, W) B(x-\beta \mid V, W) d x \\
& =\sum_{\alpha \in \mathbb{Z}^{n}} b_{X, W}(\alpha) \int_{\mathbb{R}^{n}} B(x-(\alpha-\beta) \mid V, W) B(x \mid V, W) d x \\
& =\sum_{\alpha \in \mathbb{Z}^{n}} b_{X, W}(\alpha) \int_{\mathbb{R}^{n}} B\left(x \mid-V, W^{-}\right) B((\beta-\alpha)-x \mid V, W) d x \\
& =\sum_{\alpha \in \mathbb{Z}^{n}} b_{X, W}(\alpha) \widetilde{B}(\beta-\alpha \mid X, W)=\Phi_{X, W}(\beta)=\delta_{0, \beta} .
\end{aligned}
$$

The inequality is proved as in the algebraic case. tion.

Because of this lemma we call the function $B_{V, W}^{*}$ the biorthogonal func-

Using the Jensen inequality and Lemma 9 we may prove (see [6]) the following

THeOREM 5. Let the family $V$ be admissible and satisfy the condition (6). Then for every $1 \leq p \leq \infty$ there exist constants $C_{1}>0$ and $C_{2}>0$ such that for every sequence $a=\left\{a_{\alpha}\right\} \subset l^{p}$,

$$
C_{1}\|a\|_{l^{p}} \leq\left\|\sum_{\alpha \in \mathbb{Z}^{n}} a_{\alpha} B(x-\alpha \mid V, W)\right\|_{L^{p}} \leq C_{2}\|a\|_{l^{p}} .
$$

COROLlary. Let the family $V$ be admissible and satisfy the condition (6). Then the system of box splines $\{B(\cdot-\alpha \mid V, W)\}_{\alpha \in \mathbb{Z}^{n}}$ is linearly independent.

The results of this paper were announced by the author in [13] and [14].

Problem. Prove that if $V_{s}$ is unimodular then $V_{s}$ satisfies (6).

\section{References}

[1] B. D. Bojanov, H. A. Hakopian, and A. A. Sahakian, Spline Functions and Multivariate Interpolations, Kluwer, 1993.

[2] C. de Boor and R. De Vore, Approximation by smooth multivariate splines, Trans. Amer Math. Soc. 276 (1983), 775-788. 
[3] C. de Boor and K. Höllig, B-splines from parallelepipeds, J. Anal. Math. 42 (1982/83), 99-115.

[4] C. de Boor, K. Höllig, and S. Riemenschneider, Box Splines, Springer, 1993.

[5] C. de Boor, R. De Vore, and A. Ron, On the construction of multivariate (pre)wavelets, Constr. Approx. 9(1993), 123-166.

[6] K. Dziedziul, Box Splines, Wyd. P.G., Gdańsk, 1997 (in Polish).

[7] G. Fix and G. Strang, A Fourier analysis of the finite element variational method, in: Constructive Aspects of Functional Analysis, G. Geymonat (ed.), Cremonese, Rome, 1973, 793-840.

[8] K. Jetter, Multivariate approximation from the cardinal interpolation point of view, in: Approximation Theory VII (Austin, TX, 1992), E. W. Cheney, C. K. Chui, and L. L. Schumaker (eds.), Academic Press, 1993, 131-161.

[9] S. Karlin and W. J. Studden, Tchebysheff Systems: with Applications in Analysis and Statistics, Interscience, New York, 1966.

[10] J. K. Kowalski, Application of box splines to the approximation of Sobolev spaces, J. Approx. Theory 61 (1990), 55-73.

[11] E. M. Stein and G. Weiss, Introduction to Fourier Analysis on Euclidean Spaces, Princeton Univ. Press, Princeton, 1971.

[12] Z. Wronicz, Chebyshevian splines, Dissertationes Math. 305 (1990).

[13] - On some generalization of box splines, Preprint 34 (January 97), Instytut Matematyki AGH.

[14] -, On some properties of box splines, Preprint 25 (January 98), WydziałMatematyki Stosowanej AGH.

Faculty of Applied Mathematics

Academy of Mining and Metallurgy

Al. Mickiewicza 30

30-059 Kraków, Poland

E-mail: wronicz@uci.agh.edu.pl 